\title{
A GEOMETRIC PROOF OF THE LEBESGUE DIFFERENTIATION THEOREM
}

\author{
DONALD AUSTIN ${ }^{1}$
}

We prove the Lebesgue differentiation theorem using only the most elementary concepts of measure theory. The proof is an instance of a general method of attack which has yielded a number of probabilistic limit theorems.

Preliminaries. We consider a real-valued function $f(x)$ on an interval $[a, b]$; Lebesgue measure on $[a, b]$ will be denoted by $\mu$. If $g_{1}$ and $\mathcal{G}_{2}$ are collections of intervals then $\mu \mathcal{G}_{1}$ is the measure of the point set covered by $G_{1}$, while $G_{1}-G_{2}$ is the collection of intervals in $G_{1}$ which are not in $g_{2}$. The notation $f(c, d)$ will mean the slope of the chord connecting $(c, f(c))$ with $(d, f(d))$. If $p$ is a partition, that is, a finite set of points containing $a$ and $b$, then $\pi(x)$ is the polygonal approximation to $f(x)$ on $p$. We assume that $f$ is rectifiable or, equivalently, of bounded variation (i.e., $l f=l u b_{\pi} l \pi<\infty$, where $l \pi$ is the length of the graph of $\pi$ ); thus $f$ is continuous except on a countable set. The upper (lower) right (left) derivates are measurable; in fact, at points of continuity of $f$, we have for the upper right derivate:

$$
f^{+}(x)=\lim _{n \rightarrow \infty} \operatorname{lub}_{0<r_{i}<1 / n} f\left(x, r_{i}\right),
$$

where $r_{i}$ is an ordering of the rationals.

Theorem. For $f(x)$ we have (i) the derivative exists a.e. and (ii) the derivative is finite a.e.

We note two elementary lemmas:

Lemma 1. Any finite collection of intervals $\mathcal{G}$ contains a disjoint subcollection $\mathrm{G}_{1}$ such that $\mu \mathrm{G}_{1} \geqq(1 / 3) \mu \mathrm{g}$.

Proof. Let $I_{1}$ be an interval of $\mathcal{G}$ of maximal length and $\left\{I_{1}\right\}$ the intervals of $\mathcal{G}$ which intersect $I_{1}$. Inductively, $I_{j}$ is an interval of $\mathcal{G}-\left\{\left\{I_{1}\right\}, \cdots,\left\{I_{j-1}\right\}\right\}$ of maximal length and $\left\{I_{j}\right\}$ the intervals intersecting $I_{j}$. There is a $k$ such that $\mathcal{G}=\left\{\left\{I_{1}\right\}, \cdots,\left\{I_{k}\right\}\right\}$ and then $G_{1}=\left\{I_{1}, \cdots, I_{k}\right\}$ has the desired properties.

LEMmA 2. If $\pi(x)$ is linear on $[a, b]$ with $\pi(a) \leqq \pi(b)$ and if $q(x)$ is $a$ polygon coinciding with $\pi$ at $a$ and $b$ and such that $q\left(c_{i}, d_{i}\right)<-\alpha$

Received by the editors July 10, 1962 and, in revised form, August 22, 1963.

1 Research supported by U. S. Air Force under AFOSR 62-350. 
$(\alpha>0)$, where $\left[c_{i}, d_{i}\right]$ is a finite disjoint collection of intervals the sum of whose lengths is $d$, then $l q>l \pi+d\left[\sqrt{ }\left(1+a^{2}\right)-1\right]$.

Proof. By translations of the sides of $q$ parallel to the coordinate axes we obtain an auxiliary polygon $q_{1}$ coinciding with $q$ at $a$ and $b$ and with $l q_{1}=l q$ and $q_{1}(a, a+d)<-\alpha$. Let $q_{2}$ and $q_{3}$ be the two-sided polygons determined by the endpoints of $q$ and $(a+d, q(a+d))$ and $(a+d, q(a))$, respectively. Then the following relation is obvious and establishes the lemma:

$$
\begin{aligned}
l \pi & \leqq l q_{3}<l q_{2}-d\left[\sqrt{ }\left(1+\alpha^{2}\right)-1\right]<l q_{1}-d\left[\sqrt{ }\left(1+\alpha^{2}\right)-1\right] \\
& \leqq l q-d\left[\sqrt{ }\left(1+\alpha^{2}\right)-1\right] .
\end{aligned}
$$

Proof of Theorem. Suppose (i) is not true, then there must be numbers $\beta$ and $\alpha>0$ such that if $E$ is the set where $f$ is continuous and $f^{+}>\beta+\alpha, f_{-}<\beta-\alpha$ then $\mu E>0$. Since we may add a linear function, it is no restriction to assume that $\beta=0$. Let $\pi(x)$ be any polygonal approximation to $f(x)$. We may cover each point $x \in E-p$ with an open interval $\left(a_{x}, b_{x}\right)$ such that $p$ is linear on $\left[a_{x}, b_{x}\right]$ and $f\left(a_{x}, b_{x}\right)$ $<-\alpha,>\alpha$, according as $\pi\left(a_{x}, b_{x}\right) \geqq 0$, $\leqq 0$. Using the Lindelöf theorem we may pick a finite collection $\mathcal{G}$ of the $\left(a_{x}, b_{x}\right)$ such that

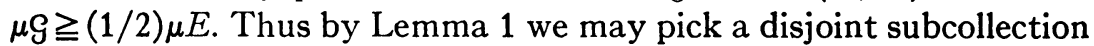
$G_{1}$ such that $\mu G_{1} \geqq(1 / 6) \mu E$. Finally by Lemma 2 we see that the polygon $q$, determined by the partition consisting of $p$ and the endpoints of the intervals of $\mathcal{G}_{1}$, satisfies $l q>l \pi+(1 / 6) \mu E\left[\sqrt{ }\left(1+a^{2}\right)-1\right]$ and hence $l f=\infty$, contradicting that $f$ is of bounded variation.

Thus $f^{+} \leqq f_{-}$and (i) follows at once; to verify (ii) we again use an indirect argument and assume that on a set $E$ as above, $f^{+}=+\infty$, then for any $M>0$ we may pick the covering $\left(a_{x}, b_{x}\right)$ of $E$ so that $f\left(a_{x}, b_{x}\right)>M$. We pick $\mathcal{G}_{1}$ as before and let $q$ be the partition determined by the endpoints of $\mathcal{G}_{1}$; then clearly $l q>d \sqrt{ }\left(1+M^{2}\right)$ and since $M$ is arbitrary, we again get a contradiction. This completes the proof.

NORTHWESTERN UNIVERSTTY 